Acta Crystallographica Section E

Structure Reports

Online

ISSN 1600-5368

José M. de la Torre, ${ }^{\mathrm{a}}$ Justo Cobo, ${ }^{\mathrm{a}}$

Manuel Nogueras ${ }^{\mathrm{a}}$ and

John Nicolson Low ${ }^{b}$ *

a Departamento de Química Inorgánica y Orgánica, Universidad de Jaén, 23071 Jaén, Spain, and ${ }^{\mathbf{b}}$ Department of Chemistry,

University of Aberdeen, Meston Walk, Old

Aberdeen, AB24 3UE, Scotland.

Correspondence e-mail: che562@abdn.ac.uk

\section{Key indicators}

Single-crystal X-ray study

$T=120 \mathrm{~K}$

Mean $\sigma(\mathrm{C}-\mathrm{C})=0.003 \AA$

$R$ factor $=0.040$

$w R$ factor $=0.106$

Data-to-parameter ratio $=18.3$

For details of how these key indicators were automatically derived from the article, see http://journals.iucr.org/e.

\title{
6-Chloro-4-(dimethylaminomethyleneamino)- 2-(methylsulfanyl)pyrimidine
}

The molecules in the title compound, $\mathrm{C}_{8} \mathrm{H}_{11} \mathrm{ClN}_{4} \mathrm{~S}$, are linked in pairs by a $\pi-\pi$ stacking interaction. There are, however, no other direction-specific interactions.

\section{Comment}

In our search for good candidates for intermediates in the synthesis of new pyrimidine fused ring systems, we have prepared the title compound, (I), (Fig. 1), a formyl derivative of 4-amino-6-chloro-2-(methylsulfanyl)pyrimidine, using the Vilsmeier formylation reaction (Vilsmeier \& Haack, 1927).

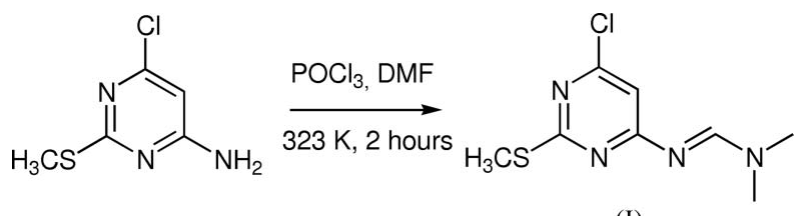

(I)

The bond lengths and angles show no unusual features. The essentially planar group consisting of atoms N4, C41, N42, C43 and $\mathrm{C} 44$ forms a dihedral angle of $31.49(8)^{\circ}$ with that of the planar pyrimidine ring. The leading torsion angles are given in Table 1 . The molecules are linked into pairs by a $\pi-\pi$ stacking

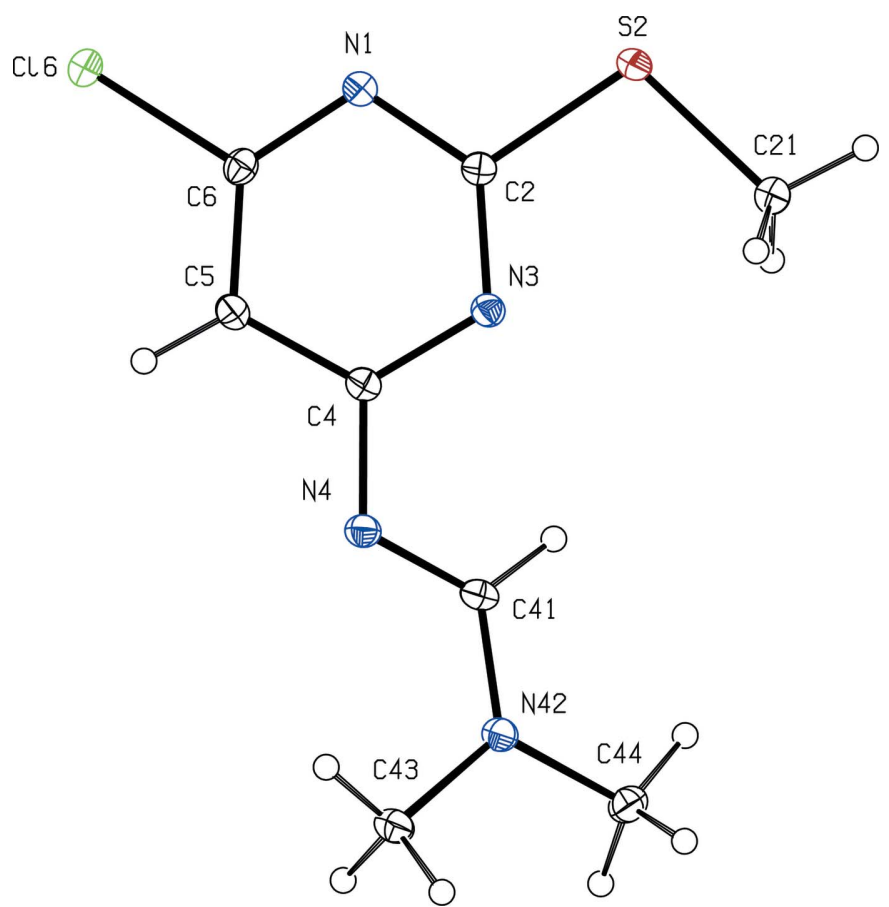

Figure 1

A view of (I) with our numbering scheme. Displacement ellipsoids are drawn at the $30 \%$ probability level.
Received 24 July 2006 Accepted 14 August 2006
(C) 2006 International Union of Crystallography All rights reserved 
interaction (Fig. 2). The molecules at $(x, y, z)$ and $(1-x, 1-y$, $1-z$ ) are parallel, with an interplanar spacing of 3.4661 (2) $⿱$ A. The ring-centroid separation is 3.359 (2) A corresponding to a ring offset of $0.857 \AA$.

\section{Experimental}

The Vilsmeier reagent was prepared in an ice-bath by adding phosphorus oxychloride $(1.8 \mathrm{mmol})$ to $N, N$-dimethylformamide (38 mmol) and stirring for $15 \mathrm{~min}$. 4-Amino-6-chloro-2-(methylsulfanyl)pyrimidine $(0.2 \mathrm{~g}, 1.14 \mathrm{mmol})$ was then added and the reaction temperature raised to $323-333 \mathrm{~K}$, and the mixture stirred for $2 \mathrm{~h}$. The reaction mixture was then poured on to crushed ice and neutralized with $\mathrm{NaOH}$ (10\% in water) until the $\mathrm{pH}$ was raised to $8-$ 9. The resulting white solid was filtered off and recrystallized from DMSO- $d_{6}$ producing white crystalline blocks suitable for singlecrystal X-ray diffraction (yield 60\%; m.p. 374-376 K). MS (70 eV): 232/230 (38:100, $\left.M+2 / M^{+}\right), 217 / 215\left(17 / 18,\left[(M+2 / M)-\mathrm{CH}_{3}\right]^{+}\right), 186 /$ $184\left(17 / 18,\left[(M+2 / M)-\mathrm{SCH}_{2}\right]^{+}\right), 149\left(31,\left[M-\mathrm{SCH}_{3}-\mathrm{Cl}\right]^{+}\right), 71$ $\left(4,\left[\mathrm{~N}=\mathrm{CH}-\mathrm{N}\left(\mathrm{CH}_{3}\right)_{2}\right]^{+}\right)$.

\section{Crystal data}

$$
\begin{aligned}
& \mathrm{C}_{8} \mathrm{H}_{11} \mathrm{ClN}_{4} \mathrm{~S} \\
& M_{r}=230.72 \\
& \text { Triclinic, } P \overline{1} \\
& a=7.4817(2) \AA \\
& b=8.5739(2) \AA \\
& c=9.818(3) \AA \\
& \alpha=111.973(2)^{\circ} \\
& \beta=91.661(2)^{\circ} \\
& \gamma=114.566(2)^{\circ}
\end{aligned}
$$

$$
\begin{aligned}
& V=518.31(15) \AA^{3} \\
& Z=2 \\
& D_{x}=1.478 \mathrm{Mg} \mathrm{m}^{-3} \\
& \text { Mo } K \alpha \text { radiation }^{-1} \\
& \mu=0.54 \mathrm{~mm}^{-1} \\
& T=120(2) \mathrm{K} \\
& \text { Block, colourless } \\
& 0.30 \times 0.30 \times 0.20 \mathrm{~mm}
\end{aligned}
$$

Data collection

Nonius KappaCCD diffractometer $\varphi$ and $\omega$ scans

Absorption correction: multi-scan (SADABS; Sheldrick, 2003)

$T_{\min }=0.856, T_{\max }=0.901$

\section{Refinement}

Refinement on $F^{2}$

$R\left[F^{2}>2 \sigma\left(F^{2}\right)\right]=0.040$

$w R\left(F^{2}\right)=0.106$

$S=1.13$

2378 reflections

130 parameters

$\mathrm{H}$-atom parameters constrained

$$
\begin{gathered}
w=1 /\left[\sigma^{2}\left(F_{\mathrm{o}}^{2}\right)+(0.0456 P)^{2}\right. \\
+0.5109 P] \\
\text { where } P=\left(F_{\mathrm{o}}^{2}+2 F_{\mathrm{c}}^{2}\right) / 3 \\
(\Delta / \sigma)_{\max }<0.001 \\
\Delta \rho_{\max }=0.43 \mathrm{e} \AA^{-3} \\
\Delta \rho_{\min }=-0.36 \mathrm{e} \AA^{-3}
\end{gathered}
$$

12192 measured reflections 378 independent reflections $R_{\text {int }}=0.032$ $\theta_{\max }=27.5^{\circ}$

Table 1

Selected torsion angles $\left({ }^{\circ}\right)$.

\begin{tabular}{lclr}
\hline $\mathrm{N} 3-\mathrm{C} 2-\mathrm{S} 2-\mathrm{C} 21$ & $0.17(18)$ & $\mathrm{N} 4-\mathrm{C} 41-\mathrm{N} 41-\mathrm{C} 43$ & \multicolumn{1}{c}{$-3.4(3)$} \\
$\mathrm{N} 1-\mathrm{C} 2-\mathrm{S} 2-\mathrm{C} 21$ & $-179.75(14)$ & $\mathrm{N} 4-\mathrm{C} 41-\mathrm{N} 41-\mathrm{C} 44$ & $175.22(18)$ \\
$\mathrm{N} 3-\mathrm{C} 4-\mathrm{N} 4-\mathrm{C} 41$ & $-25.4(3)$ & $\mathrm{C} 2-\mathrm{N} 1-\mathrm{C} 6-\mathrm{Cl} 6$ & $-177.26(13)$ \\
$\mathrm{C} 5-\mathrm{C} 4-\mathrm{N} 4-\mathrm{C} 41$ & $156.38(18)$ & $\mathrm{C} 4-\mathrm{C} 5-\mathrm{C} 6-\mathrm{Cl} 6$ & $175.75(14)$ \\
$\mathrm{C} 4-\mathrm{N} 4-\mathrm{C} 41-\mathrm{N} 41$ & $174.23(17)$ & & \\
\hline
\end{tabular}

$\mathrm{H}$ atoms were treated as riding atoms, with aromatic $\mathrm{C}-\mathrm{H}=$ $0.95 \AA$ and $U_{\text {iso }}(\mathrm{H})=1.2 U_{\text {eq }}(\mathrm{C})$, and $\mathrm{C}-\mathrm{H}=0.98 \AA$ and $U_{\text {iso }}(\mathrm{H})=$ $1.5 U_{\text {eq }}(\mathrm{C})$. The positions of all methyl $\mathrm{H}$ atoms were checked in a difference map.

Data collection: COLLECT (Bruker-Nonius, 2004); cell refinement: DIRAX/LSQ (Duisenberg et al., 2000); data reduction:

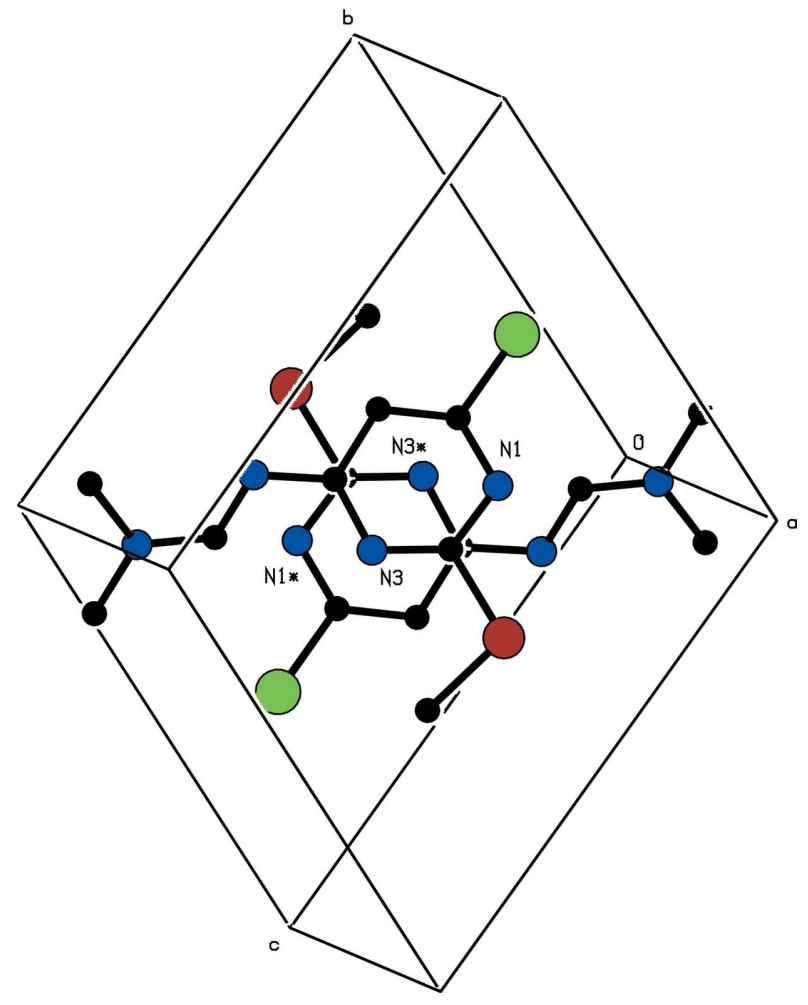

Figure 2

A view of the $\pi-\pi$ stacking viewed perpendicular to the plane of the pyrimidine ring. Atoms labelled with an asterisk $(*)$ are in the molecule at $(1-x, 1-y, 1-z)$. For the sake of clarity, all $\mathrm{H}$ atoms have been omitted.

EVALCCD (Duisenberg et al., 2003); program(s) used to solve structure: SIR2004 (Burla et al., 2005); program(s) used to refine structure: OSCAIL (McArdle, 2003) and SHELXL97 (Sheldrick, 1997); molecular graphics: PLATON (Spek, 2003); software used to prepare material for publication: SHELXL97 and WORDPERFECT macro PRPKAPPA (Ferguson, 1999).

JT, MN and JC thank the Consejería de Innovacíon, Ciencia $y$ Empresa (Junta de Andalucía, Spain) and the Universidad de Jaén, Spain, for financial support. JT thanks also the Universidad de Jaén for a research scholarship.

\section{References}

Bruker-Nonius (2004). COLLECT. Bruker-Nonius BV, Delft, The Netherlands.

Burla, M. C., Caliandro, R., Camalli, M., Carrozzini, B., Cascarano, G. L., De Caro, L., Giacovazzo, C., Polidori, G. \& Spagna, R. (2005). J. Appl. Cryst. 38, 381-388.

Duisenberg, A. J. M, Hooft, R. W. W., Schreurs, A. M. M. \& Kroon, J. (2000). J. Appl. Cryst. 33, 893-898.

Duisenberg, A. J. M., Kroon-Batenburg, L. M. J. \& Schreurs, A. M. M. (2003). J. Appl. Cryst. 36, 220-229.

Ferguson, G. (1999). PRPKAPPA. University of Guelph, Canada.

McArdle, P. (2003). OSCAIL for Windows. Version 10. Crystallography Centre, Chemistry Department, NUI Galway, Ireland.

Sheldrick, G. M. (1997). SHELXL97. University of Göttingen, Germany.

Sheldrick, G. M. (2003). SADABS. Version 2.10. University of Göttingen, Germany.

Spek, A. L. (2003). J. Appl. Cryst. 36, 7-13.

Vilsmeier, A. \& Haack, A. (1927). Chem. Ber. 60, 119-122. 


\section{supporting information}

Acta Cryst. (2006). E62, o3910-o3911 [https://doi.org/10.1107/S1600536806032004]

\section{6-Chloro-4-(dimethylaminomethyleneamino)-2-(methylsulfanyl)pyrimidine}

José M. de la Torre, Justo Cobo, Manuel Nogueras and John Nicolson Low

6-Chloro-4-(dimethylaminomethyleneamino)-2-(methylsulfanyl)pyrimidine

Crystal data

$\mathrm{C}_{8} \mathrm{H}_{11} \mathrm{ClN}_{4} \mathrm{~S}$

$M_{r}=230.72$

Triclinic, $P \overline{1}$

$a=7.4817$ (2) $\AA$

$b=8.5739(2) \AA$

$c=9.818(3) \AA$

$\alpha=111.973(2)^{\circ}$

$\beta=91.661(2)^{\circ}$

$\gamma=114.566(2)^{\circ}$

$V=518.31(15) \AA^{3}$

$$
\begin{aligned}
& Z=2 \\
& F(000)=240
\end{aligned}
$$

$D_{\mathrm{x}}=1.478 \mathrm{Mg} \mathrm{m}^{-3}$

Mo $K \alpha$ radiation, $\lambda=0.71069 \AA$

Cell parameters from 2378 reflections

$\theta=4.2-27.5^{\circ}$

$\mu=0.54 \mathrm{~mm}^{-1}$

$T=120 \mathrm{~K}$

Block, colourless

$0.30 \times 0.30 \times 0.20 \mathrm{~mm}$

Data collection

Nonius KappaCCD

2378 independent reflections

diffractometer

$\varphi$ and $\omega$ scans

Absorption correction: multi-scan

(SADABS; Sheldrick, 2003)

$T_{\min }=0.856, T_{\max }=0.901$

12192 measured reflections

2015 reflections with $I>2 \sigma(I)$

$R_{\text {int }}=0.032$

$\theta_{\max }=27.5^{\circ}, \theta_{\min }=4.2^{\circ}$

$h=-9 \rightarrow 9$

$k=-11 \rightarrow 11$

$l=-12 \rightarrow 12$

Refinement

Refinement on $F^{2}$

Least-squares matrix: full

$R\left[F^{2}>2 \sigma\left(F^{2}\right)\right]=0.040$

$w R\left(F^{2}\right)=0.106$

$S=1.13$

2378 reflections

130 parameters

0 restraints

Primary atom site location: structure-invariant

direct methods

Secondary atom site location: difference Fourier map

Hydrogen site location: inferred from neighbouring sites

$\mathrm{H}$-atom parameters constrained

$w=1 /\left[\sigma^{2}\left(F_{\mathrm{o}}^{2}\right)+(0.0456 P)^{2}+0.5109 P\right]$ where $P=\left(F_{\mathrm{o}}^{2}+2 F_{\mathrm{c}}^{2}\right) / 3$

$(\Delta / \sigma)_{\max }<0.001$

$\Delta \rho_{\max }=0.43$ e $\AA^{-3}$

$\Delta \rho_{\min }=-0.36$ e $\AA^{-3}$

Special details

Experimental. The scale factors in the experimental table are calculated from the 'size' command in the SHELXL97 input file. 
Fractional atomic coordinates and isotropic or equivalent isotropic displacement parameters $\left(\AA^{2}\right)$

\begin{tabular}{lllll}
\hline & $x$ & $y$ & $z$ & $U_{\text {iso }} * U_{\text {eq }}$ \\
\hline $\mathrm{N} 1$ & $0.7202(2)$ & $0.4345(2)$ & $0.35311(18)$ & $0.0187(3)$ \\
$\mathrm{C} 2$ & $0.7336(3)$ & $0.4427(3)$ & $0.4933(2)$ & $0.0171(4)$ \\
$\mathrm{S} 2$ & $0.71334(8)$ & $0.23243(7)$ & $0.49580(5)$ & $0.02132(15)$ \\
$\mathrm{C} 21$ & $0.7347(3)$ & $0.2824(3)$ & $0.6919(2)$ & $0.0255(4)$ \\
$\mathrm{N} 3$ & $0.7589(2)$ & $0.5869(2)$ & $0.62132(18)$ & $0.0176(3)$ \\
$\mathrm{C} 4$ & $0.7747(3)$ & $0.7458(3)$ & $0.6112(2)$ & $0.0172(4)$ \\
$\mathrm{N} 4$ & $0.8032(2)$ & $0.9024(2)$ & $0.73831(18)$ & $0.0192(3)$ \\
$\mathrm{C} 41$ & $0.7393(3)$ & $0.8703(3)$ & $0.8511(2)$ & $0.0183(4)$ \\
$\mathrm{N} 41$ & $0.7704(2)$ & $1.0073(2)$ & $0.98542(18)$ & $0.0195(3)$ \\
$\mathrm{C} 43$ & $0.8898(3)$ & $1.2062(3)$ & $1.0181(2)$ & $0.0241(4)$ \\
$\mathrm{C} 44$ & $0.6831(3)$ & $0.9661(3)$ & $1.1064(2)$ & $0.0234(4)$ \\
$\mathrm{C} 5$ & $0.7678(3)$ & $0.7549(3)$ & $0.4712(2)$ & $0.0191(4)$ \\
$\mathrm{C} 6$ & $0.7360(3)$ & $0.5939(3)$ & $0.3494(2)$ & $0.0185(4)$ \\
$\mathrm{C} 66$ & $0.70810(8)$ & $0.58526(7)$ & $0.16889(5)$ & $0.02420(15)$ \\
$\mathrm{H} 21 \mathrm{~A}$ & 0.7244 & 0.1718 & 0.7059 & $0.038^{*}$ \\
$\mathrm{H} 21 \mathrm{~B}$ & 0.6262 & 0.3111 & 0.7275 & $0.038^{*}$ \\
$\mathrm{H} 21 \mathrm{C}$ & 0.8652 & 0.3914 & 0.7495 & $0.038^{*}$ \\
$\mathrm{H} 41$ & 0.6665 & 0.7426 & 0.8370 & $0.022^{*}$ \\
$\mathrm{H} 43 \mathrm{~A}$ & 0.9684 & 1.2170 & 0.9409 & $0.036^{*}$ \\
$\mathrm{H} 43 \mathrm{~B}$ & 0.9814 & 1.2724 & 1.1170 & $0.036^{*}$ \\
$\mathrm{H} 43 \mathrm{C}$ & 0.8002 & 1.2632 & 1.0183 & $0.036^{*}$ \\
$\mathrm{H} 44 \mathrm{~A}$ & 0.5920 & 0.8302 & 1.0680 & $0.035^{*}$ \\
$\mathrm{H} 44 \mathrm{~B}$ & 0.6078 & 1.0377 & 1.1441 & $0.035^{*}$ \\
$\mathrm{H} 44 \mathrm{C}$ & 0.7910 & 1.0027 & 1.1885 & $0.035^{*}$ \\
$\mathrm{H} 5$ & 0.7842 & 0.8662 & 0.4618 & $0.023^{*}$ \\
& & & &
\end{tabular}

Atomic displacement parameters $\left(\AA^{2}\right)$

\begin{tabular}{lllllll}
\hline & $U^{11}$ & $U^{22}$ & $U^{33}$ & $U^{12}$ & $U^{13}$ & $U^{23}$ \\
\hline $\mathrm{N} 1$ & $0.0197(8)$ & $0.0177(8)$ & $0.0186(8)$ & $0.0086(7)$ & $0.0043(6)$ & $0.0077(6)$ \\
$\mathrm{C} 2$ & $0.0159(9)$ & $0.0168(9)$ & $0.0173(9)$ & $0.0072(7)$ & $0.0030(7)$ & $0.0064(7)$ \\
$\mathrm{S} 2$ & $0.0301(3)$ & $0.0171(2)$ & $0.0182(3)$ & $0.0133(2)$ & $0.00435(19)$ & $0.00624(19)$ \\
$\mathrm{C} 21$ & $0.0362(12)$ & $0.0228(10)$ & $0.0195(10)$ & $0.0149(9)$ & $0.0050(8)$ & $0.0094(8)$ \\
$\mathrm{N} 3$ & $0.0191(8)$ & $0.0167(8)$ & $0.0170(8)$ & $0.0088(6)$ & $0.0024(6)$ & $0.0065(6)$ \\
C4 & $0.0153(8)$ & $0.0160(9)$ & $0.0185(9)$ & $0.0069(7)$ & $0.0023(7)$ & $0.0062(7)$ \\
N4 & $0.0217(8)$ & $0.0176(8)$ & $0.0172(8)$ & $0.0098(7)$ & $0.0023(6)$ & $0.0055(6)$ \\
C41 & $0.0179(9)$ & $0.0154(9)$ & $0.0188(9)$ & $0.0087(7)$ & $0.0017(7)$ & $0.0036(7)$ \\
N41 & $0.0220(8)$ & $0.0178(8)$ & $0.0184(8)$ & $0.0100(7)$ & $0.0032(6)$ & $0.0064(7)$ \\
C43 & $0.0299(11)$ & $0.0174(9)$ & $0.0222(10)$ & $0.0112(8)$ & $0.0031(8)$ & $0.0054(8)$ \\
C44 & $0.0266(10)$ & $0.0270(10)$ & $0.0187(9)$ & $0.0146(9)$ & $0.0064(8)$ & $0.0091(8)$ \\
C5 & $0.0203(9)$ & $0.0163(9)$ & $0.0210(9)$ & $0.0084(8)$ & $0.0040(7)$ & $0.0084(8)$ \\
C6 & $0.0174(9)$ & $0.0222(9)$ & $0.0174(9)$ & $0.0097(8)$ & $0.0040(7)$ & $0.0093(8)$ \\
C16 & $0.0333(3)$ & $0.0244(3)$ & $0.0183(2)$ & $0.0146(2)$ & $0.00694(19)$ & $0.0109(2)$ \\
& & & & & &
\end{tabular}


Geometric parameters $\left(\AA,{ }^{\circ}\right)$

\begin{tabular}{|c|c|c|c|}
\hline $\mathrm{N} 1-\mathrm{C} 6$ & $1.337(2)$ & $\mathrm{C} 41-\mathrm{H} 41$ & 0.95 \\
\hline $\mathrm{N} 1-\mathrm{C} 2$ & $1.350(2)$ & $\mathrm{N} 41-\mathrm{C} 43$ & $1.455(3)$ \\
\hline $\mathrm{C} 2-\mathrm{N} 3$ & $1.331(2)$ & $\mathrm{N} 41-\mathrm{C} 44$ & $1.460(3)$ \\
\hline $\mathrm{C} 2-\mathrm{S} 2$ & $1.7553(19)$ & $\mathrm{C} 43-\mathrm{H} 43 \mathrm{~A}$ & 0.98 \\
\hline $\mathrm{S} 2-\mathrm{C} 21$ & $1.796(2)$ & $\mathrm{C} 43-\mathrm{H} 43 \mathrm{~B}$ & 0.98 \\
\hline $\mathrm{C} 21-\mathrm{H} 21 \mathrm{~A}$ & 0.98 & $\mathrm{C} 43-\mathrm{H} 43 \mathrm{C}$ & 0.98 \\
\hline $\mathrm{C} 21-\mathrm{H} 21 \mathrm{~B}$ & 0.98 & $\mathrm{C} 44-\mathrm{H} 44 \mathrm{~A}$ & 0.98 \\
\hline $\mathrm{C} 21-\mathrm{H} 21 \mathrm{C}$ & 0.98 & $\mathrm{C} 44-\mathrm{H} 44 \mathrm{~B}$ & 0.98 \\
\hline $\mathrm{N} 3-\mathrm{C} 4$ & $1.360(2)$ & $\mathrm{C} 44-\mathrm{H} 44 \mathrm{C}$ & 0.98 \\
\hline $\mathrm{C} 4-\mathrm{N} 4$ & $1.378(2)$ & $\mathrm{C} 5-\mathrm{C} 6$ & $1.367(3)$ \\
\hline $\mathrm{C} 4-\mathrm{C} 5$ & $1.406(3)$ & $\mathrm{C} 5-\mathrm{H} 5$ & 0.95 \\
\hline $\mathrm{N} 4-\mathrm{C} 41$ & $1.296(3)$ & $\mathrm{C} 6-\mathrm{Cl} 6$ & $1.748(2)$ \\
\hline $\mathrm{C} 41-\mathrm{N} 41$ & $1.330(2)$ & & \\
\hline $\mathrm{C} 6-\mathrm{N} 1-\mathrm{C} 2$ & $113.18(16)$ & $\mathrm{C} 41-\mathrm{N} 41-\mathrm{C} 44$ & $121.80(17)$ \\
\hline $\mathrm{N} 3-\mathrm{C} 2-\mathrm{N} 1$ & $127.66(17)$ & $\mathrm{C} 43-\mathrm{N} 41-\mathrm{C} 44$ & $116.60(16)$ \\
\hline $\mathrm{N} 3-\mathrm{C} 2-\mathrm{S} 2$ & $119.89(14)$ & $\mathrm{N} 41-\mathrm{C} 43-\mathrm{H} 43 \mathrm{~A}$ & 109.5 \\
\hline $\mathrm{N} 1-\mathrm{C} 2-\mathrm{S} 2$ & $112.44(14)$ & $\mathrm{N} 41-\mathrm{C} 43-\mathrm{H} 43 \mathrm{~B}$ & 109.5 \\
\hline $\mathrm{C} 2-\mathrm{S} 2-\mathrm{C} 21$ & $102.86(9)$ & $\mathrm{H} 43 \mathrm{~A}-\mathrm{C} 43-\mathrm{H} 43 \mathrm{~B}$ & 109.5 \\
\hline $\mathrm{S} 2-\mathrm{C} 21-\mathrm{H} 21 \mathrm{~A}$ & 109.5 & $\mathrm{~N} 41-\mathrm{C} 43-\mathrm{H} 43 \mathrm{C}$ & 109.5 \\
\hline $\mathrm{S} 2-\mathrm{C} 21-\mathrm{H} 21 \mathrm{~B}$ & 109.5 & $\mathrm{H} 43 \mathrm{~A}-\mathrm{C} 43-\mathrm{H} 43 \mathrm{C}$ & 109.5 \\
\hline $\mathrm{H} 21 \mathrm{~A}-\mathrm{C} 21-\mathrm{H} 21 \mathrm{~B}$ & 109.5 & $\mathrm{H} 43 \mathrm{~B}-\mathrm{C} 43-\mathrm{H} 43 \mathrm{C}$ & 109.5 \\
\hline $\mathrm{S} 2-\mathrm{C} 21-\mathrm{H} 21 \mathrm{C}$ & 109.5 & $\mathrm{~N} 41-\mathrm{C} 44-\mathrm{H} 44 \mathrm{~A}$ & 109.5 \\
\hline $\mathrm{H} 21 \mathrm{~A}-\mathrm{C} 21-\mathrm{H} 21 \mathrm{C}$ & 109.5 & $\mathrm{~N} 41-\mathrm{C} 44-\mathrm{H} 44 \mathrm{~B}$ & 109.5 \\
\hline $\mathrm{H} 21 \mathrm{~B}-\mathrm{C} 21-\mathrm{H} 21 \mathrm{C}$ & 109.5 & $\mathrm{H} 44 \mathrm{~A}-\mathrm{C} 44-\mathrm{H} 44 \mathrm{~B}$ & 109.5 \\
\hline $\mathrm{C} 2-\mathrm{N} 3-\mathrm{C} 4$ & $116.74(16)$ & $\mathrm{N} 41-\mathrm{C} 44-\mathrm{H} 44 \mathrm{C}$ & 109.5 \\
\hline $\mathrm{N} 3-\mathrm{C} 4-\mathrm{N} 4$ & $120.58(17)$ & $\mathrm{H} 44 \mathrm{~A}-\mathrm{C} 44-\mathrm{H} 44 \mathrm{C}$ & 109.5 \\
\hline $\mathrm{N} 3-\mathrm{C} 4-\mathrm{C} 5$ & $120.53(17)$ & $\mathrm{H} 44 \mathrm{~B}-\mathrm{C} 44-\mathrm{H} 44 \mathrm{C}$ & 109.5 \\
\hline $\mathrm{N} 4-\mathrm{C} 4-\mathrm{C} 5$ & $118.87(17)$ & $\mathrm{C} 6-\mathrm{C} 5-\mathrm{C} 4$ & $115.92(17)$ \\
\hline $\mathrm{C} 41-\mathrm{N} 4-\mathrm{C} 4$ & $116.07(17)$ & $\mathrm{C} 6-\mathrm{C} 5-\mathrm{H} 5$ & 122.0 \\
\hline $\mathrm{N} 4-\mathrm{C} 41-\mathrm{N} 41$ & $123.31(18)$ & $\mathrm{C} 4-\mathrm{C} 5-\mathrm{H} 5$ & 122.0 \\
\hline $\mathrm{N} 4-\mathrm{C} 41-\mathrm{H} 41$ & 118.3 & $\mathrm{~N} 1-\mathrm{C} 6-\mathrm{C} 5$ & $125.91(18)$ \\
\hline $\mathrm{N} 41-\mathrm{C} 41-\mathrm{H} 41$ & 118.3 & $\mathrm{~N} 1-\mathrm{C} 6-\mathrm{Cl} 6$ & $114.60(14)$ \\
\hline $\mathrm{C} 41-\mathrm{N} 41-\mathrm{C} 43$ & $121.59(17)$ & $\mathrm{C} 5-\mathrm{C} 6-\mathrm{Cl} 6$ & $119.48(15)$ \\
\hline $\mathrm{C} 6-\mathrm{N} 1-\mathrm{C} 2-\mathrm{N} 3$ & $0.3(3)$ & $\mathrm{C} 4-\mathrm{N} 4-\mathrm{C} 41-\mathrm{N} 41$ & $174.23(17)$ \\
\hline $\mathrm{C} 6-\mathrm{N} 1-\mathrm{C} 2-\mathrm{S} 2$ & $-179.79(13)$ & $\mathrm{N} 4-\mathrm{C} 41-\mathrm{N} 41-\mathrm{C} 43$ & $-3.4(3)$ \\
\hline $\mathrm{N} 3-\mathrm{C} 2-\mathrm{S} 2-\mathrm{C} 21$ & $0.17(18)$ & $\mathrm{N} 4-\mathrm{C} 41-\mathrm{N} 41-\mathrm{C} 44$ & $175.22(18)$ \\
\hline $\mathrm{N} 1-\mathrm{C} 2-\mathrm{S} 2-\mathrm{C} 21$ & $-179.75(14)$ & $\mathrm{N} 3-\mathrm{C} 4-\mathrm{C} 5-\mathrm{C} 6$ & $2.7(3)$ \\
\hline $\mathrm{N} 1-\mathrm{C} 2-\mathrm{N} 3-\mathrm{C} 4$ & $-0.5(3)$ & $\mathrm{N} 4-\mathrm{C} 4-\mathrm{C} 5-\mathrm{C} 6$ & $-179.04(17)$ \\
\hline $\mathrm{S} 2-\mathrm{C} 2-\mathrm{N} 3-\mathrm{C} 4$ & $179.60(13)$ & $\mathrm{C} 2-\mathrm{N} 1-\mathrm{C} 6-\mathrm{C} 5$ & $1.6(3)$ \\
\hline $\mathrm{C} 2-\mathrm{N} 3-\mathrm{C} 4-\mathrm{N} 4$ & $-179.32(17)$ & $\mathrm{C} 2-\mathrm{N} 1-\mathrm{C} 6-\mathrm{Cl} 6$ & $-177.26(13)$ \\
\hline $\mathrm{C} 2-\mathrm{N} 3-\mathrm{C} 4-\mathrm{C} 5$ & $-1.1(3)$ & $\mathrm{C} 4-\mathrm{C} 5-\mathrm{C} 6-\mathrm{N} 1$ & $-3.1(3)$ \\
\hline $\mathrm{N} 3-\mathrm{C} 4-\mathrm{N} 4-\mathrm{C} 41$ & $-25.4(3)$ & $\mathrm{C} 4-\mathrm{C} 5-\mathrm{C} 6-\mathrm{Cl} 6$ & $175.75(14)$ \\
\hline $\mathrm{C} 5-\mathrm{C} 4-\mathrm{N} 4-\mathrm{C} 41$ & $156.38(18)$ & & \\
\hline
\end{tabular}

